\title{
Spectral functions and their applications
}

\author{
Valery N.Marachevsky * \\ V. A. Fock Institute of Physics, St. Petersburg University, \\ 198504 St. Petersburg, Russia
}

November 15, 2018

\begin{abstract}
We give an introduction to the heat kernel technique and $\zeta$-function. Two applications are considered. First we derive the high temperature asymptotics of the free energy for boson fields in terms of the heat kernel expansion and $\zeta$-function. Another application is chiral anomaly for local (MIT bag) boundary conditions.
\end{abstract}

\section{Introduction}

In this paper we give an introduction to the technique of the heat-kernel expansion and $\zeta$-function regularization. The heat kernel became a standard tool in calculations of the vacuum polarization, the Casimir effect and quantum anomalies. In the case of non-trivial background fields, and especially in the presence of boundaries or singularities, the heat kernel technique seems to be the most adequate one for the analysis of the one-loop effects (see [1] for a recent review).

A possible application of heat kernel methods to the evaluation of high temperature asymptotics of the free energy in the presense of boundaries or singularities has not received due attention yet. At high temperatures the expansion of the free energy can be determined by the heat kernel expansion and $\zeta$-function [2].

Chiral anomaly, which was discovered more than 30 years ago [3], still plays an important role in physics. On smooth manifolds without boundaries many successful approaches to the anomalies exist [4]. The heat kernel approach to the anomalies is essentially equivalent to the Fujikawa approach [5] and to the calculations based on the finite-mode regularization [6], but it can be more easily extended to complicated geometries. The chiral anomaly in the case of nontrivial boundary conditions (MIT bag boundary conditions) has been calculated only recently [7].

*email: maraval@mail.ru, root@VM1485.spb.edu 
The paper is organized as follows. In Sec. 2 we give an introduction to the formalism of the heat kernel and heat kernel expansion. Also we introduce a zeta function and calculate the one-loop effective action in terms of the zeta function. In Sec. 3 we consider two examples. First we derive the high temperature expansion of the free energy for boson fields in terms of the heat kernel expansion and $\zeta$-function. Then we discuss a chiral anomaly in four dimensions for an euclidean version of the MIT bag boundary conditions [8].

\section{Spectral functions}

\section{$2.1 \quad$ Heat kernel}

Consider a second order elliptic partial differential operator $L$ of Laplace type on an n-dimensional Riemannian manifold. Any operator of this type can be expanded locally as

$$
L=-\left(g^{\mu \nu} \partial_{\mu} \partial_{\nu}+a^{\sigma} \partial_{\sigma}+b\right),
$$

where $a$ and $b$ are some matrix valued functions and $g^{\mu \nu}$ is the inverse metric tensor on the manifold. For a flat space $g^{\mu \nu}=\delta^{\mu \nu}$.

The heat kernel can be defined as follows:

$$
K(t ; x ; y ; L)=\langle x|\exp (-t L)| y\rangle=\sum_{\lambda} \phi_{\lambda}^{\dagger}(x) \phi_{\lambda}(y) \exp (-t \lambda),
$$

where $\phi_{\lambda}$ is an eigenfunction of the operator $L$ with the eigenvalue $\lambda$.

It satisfies the heat equation

$$
\left(\partial_{t}+L_{x}\right) K(t ; x ; y ; L)=0
$$

with an initial condition

$$
K(0 ; x ; y ; L)=\delta(x, y) .
$$

If we consider the fields in a finite volume then it is necessary to specify boundary conditions. Different choices are possible. In section 3.1 we will consider the case of periodic boundary conditions on imaginary time coordinate, which are specific for boson fields. In section 3.2 we will study bag boundary conditions imposed on fermion fields. If the normal to the boundary component of the fermion current $\psi^{\dagger} \gamma_{n} \psi$ vanishes at the boundary, one can impose bag boundary conditions, a particular case of mixed boundary conditions. We assume given two complementary projectors $\Pi_{ \pm}, \Pi_{-}+\Pi_{+}=I$ acting on a multi component field (the eigenfunction of the operator $L$ ) at each point of the boundary and define mixed boundary conditions by the relations

$$
\left.\Pi_{-} \psi\right|_{\partial M}=0,\left.\quad\left(\nabla_{n}+S\right) \Pi_{+} \psi\right|_{\partial M}=0,
$$


where $S$ is a matrix valued function on the boundary. In other words, the components $\Pi_{-} \psi$ satisfy Dirichlet boundary conditions, and $\Pi_{+} \psi$ satisfy Robin (modified Neumann) ones.

It is convenient to define

$$
\chi=\Pi_{+}-\Pi_{-} .
$$

Let $\left\{e_{j}\right\}, j=1, \ldots, n$ be a local orthonormal frame for the tangent space to the manifold and let on the boundary $e_{n}$ be an inward pointing normal vector.

The extrinsic curvature is defined by the equation

$$
L_{a b}=\Gamma_{a b}^{n},
$$

where $\Gamma$ is the Christoffel symbol. For example, on the unit sphere $S^{n-1}$ which bounds the unit ball in $R^{n}$ the extrinsic curvature is $L_{a b}=\delta_{a b}$.

Curved space offers no complications in our approach compared to the flat case. Let $R_{\mu \nu \rho \sigma}$ be the Riemann tensor, and let $R_{\mu \nu}=R_{\mu \nu \sigma}^{\sigma}$ be the Ricci tensor. With our sign convention the scalar curvature $R=R_{\mu}^{\mu}$ is +2 on the unit sphere $S^{2}$. In flat space the Riemann and Ricci tensors are equal to zero.

One can always introduce a connection $\omega_{\mu}$ and another matrix valued function $E$ so that $L$ takes the form:

$$
L=-\left(g^{\mu \nu} \nabla_{\mu} \nabla_{\nu}+E\right)
$$

Here $\nabla_{\mu}$ is a sum of covariant Riemannian derivative with respect to metric $g_{\mu \nu}$ and connection $\omega_{\mu}$. One can, of course, express $E$ and $\omega$ in terms of $a^{\mu}, b$ and $g_{\mu \nu}:$

$$
\begin{aligned}
& \omega_{\mu}=\frac{1}{2} g_{\mu \nu}\left(a^{\nu}+g^{\rho \sigma} \Gamma_{\rho \sigma}^{\nu}\right) \\
& E=b-g^{\mu \nu}\left(\partial_{\nu} \omega_{\mu}+\omega_{\mu} \omega_{\nu}-\omega_{\rho} \Gamma_{\mu \nu}^{\rho}\right)
\end{aligned}
$$

For the future use we introduce also the field strength for $\omega$ :

$$
\Omega_{\mu \nu}=\partial_{\mu} \omega_{\nu}-\partial_{\nu} \omega_{\mu}+\left[\omega_{\mu}, \omega_{\nu}\right]
$$

The connection $\omega_{\mu}$ will be used to construct covariant derivatives. The subscript $; \mu \ldots \nu \sigma$ will be used to denote repeated covariant derivatives with the connection $\omega$ and the Christoffel connection on $M$. The subscript : $a \ldots b c$ will denote repeated covariant derivatives containing $\omega$ and the Christoffel connection on the boundary. Difference between these two covariant derivatives is measured by the extrinsic curvature (7). For example, $E_{; a b}=E_{: a b}-L_{a b} E_{; n}$.

Let us define an integrated heat kernel for a hermitian operator $L$ by the equation:

$$
K(Q, L, t):=\operatorname{Tr}(Q \exp (-t L))=\int_{M} d^{n} x \sqrt{g} \operatorname{tr}(Q(x) K(t ; x ; x ; L)),
$$


where $Q(x)$ is an hermitian matrix valued function, tr here is over matrix indices. For the boundary conditions we consider in this paper there exists an asymptotic expansion [9] as $t \rightarrow 0$ :

$$
K(Q, L, t) \simeq \sum_{k=0}^{\infty} a_{k}(Q, L) t^{(k-n) / 2}
$$

According to the general theory 9 the coefficients $a_{k}(Q, L)$ are locally computable. This means that each $a_{k}(Q, L)$ can be represented as a sum of volume and boundary integrals of local invariants constructed from $Q, \Omega, E$, the curvature tensor, and their derivatives. Boundary invariants may also include $S, L_{a b}$ and $\chi$. Total mass dimension of such invariants should be $k$ for the volume terms and $k-1$ for the boundary ones.

At the moment several coefficients of the expansion (13) are known for the case of mixed boundary conditions (5) and matrix valued function $Q$ (see [7] for details of derivation; the formula (49) for $a_{4}$ was derived in [7] with additional restrictions $L_{a b}=0$ and $\left.S=0\right)$ :

$$
\begin{aligned}
a_{0}(Q, L)= & (4 \pi)^{-n / 2} \int_{M} d^{n} x \sqrt{g} \operatorname{tr}(Q) . \\
a_{1}(Q, L)= & \frac{1}{4}(4 \pi)^{-(n-1) / 2} \int_{\partial M} d^{n-1} x \sqrt{h} \operatorname{tr}(\chi Q) . \\
a_{2}(Q, L)= & \frac{1}{6}(4 \pi)^{-n / 2}\left\{\int_{M} d^{n} x \sqrt{g} \operatorname{tr}(6 Q E+Q R)\right. \\
& \left.+\int_{\partial M} d^{n-1} x \sqrt{h} \operatorname{tr}\left(2 Q L_{a a}+12 Q S+3 \chi Q_{; n}\right)\right\} . \\
a_{3}(Q, L)= & \frac{1}{384}(4 \pi)^{-(n-1) / 2} \int_{\partial M} d^{n-1} x \sqrt{h} \operatorname{tr}\{Q(-24 E+24 \chi E \chi \\
& +48 \chi E+48 E \chi-12 \chi_{: a} \chi_{: a}+12 \chi_{: a a}-6 \chi_{: a} \chi_{: a} \chi+16 \chi R \\
& +8 \chi R_{a n a n}+192 S^{2}+96 L_{a a} S+(3+10 \chi) L_{a a} L_{b b} \\
& \left.\left.+(6-4 \chi) L_{a b} L_{a b}\right)+Q_{; n}\left(96 S+192 S^{2}\right)+24 \chi Q_{; n n}\right\} .
\end{aligned}
$$

For a scalar function $Q$ and mixed boundary conditions the coefficients $a_{4}$ and $a_{5}$ were already derived [10].

\section{$2.2 \zeta$-function}

Zeta function of an operator $L$ is defined by

$$
\zeta_{L}(s)=\sum_{\lambda} \frac{1}{\lambda^{s}}
$$


where the sum is over all eigenvalues of the operator $L$. The zeta function is related to the heat kernel by the transformation

$$
\zeta_{L}(s)=\frac{1}{\Gamma(s)} \int_{0}^{+\infty} d t t^{s-1} K(I, L, t) .
$$

Residues at the poles of the zeta function are related to the coefficients of the heat kernel expansion:

$$
a_{k}(I, L)=\operatorname{Res}_{s=(n-k) / 2}\left(\Gamma(s) \zeta_{L}(s)\right) .
$$

Here $I$ is a unit matrix with a dimension of the matrix functions $a^{\mu}, b$ in (1).From (201) it follows that

$$
a_{n}(I, L)=\zeta_{L}(0)
$$

In Euclidean four dimensional space the zero temperature one-loop path integral over the boson fields $\phi=\sum_{\lambda} C_{\lambda} \phi_{\lambda}$ can be evaluated as follows (up to a normalization factor):

$$
Z=\int d \phi e^{-\int d^{4} x \phi L \phi} \simeq \prod_{\lambda} \int \mu d C_{\lambda} e^{-\lambda C_{\lambda}^{2}} \simeq \mu^{\zeta_{L}(0)} \operatorname{det} L^{-1 / 2} .
$$

Here we introduced the constant $\mu$ with a dimension of mass in order to keep a proper dimension of the measure in the functional integral. $\zeta_{L}(0)$ can be thought of as a number of eigenvalues of the operator $L$. For the operator $L$ in the form (11) the number of eigenvalues is infinite, so $\zeta_{L}(0)$ yields a regularized value for this number.

The zero temperature one-loop effective action is defined then by

$$
\begin{aligned}
W & =-\ln Z=-\frac{1}{2} \ln \operatorname{det} L+\frac{1}{2} \zeta_{L}(0) \ln \mu^{2}=\frac{1}{2} \zeta_{L}^{\prime}(0)+\frac{1}{2} \zeta_{L}(0) \ln \mu^{2}= \\
& =\left.\frac{1}{2} \frac{\partial}{\partial s}\left(\mu^{2 s} \zeta_{L}(s)\right)\right|_{s=0}
\end{aligned}
$$

The term $\zeta_{L}(0) \ln \mu^{2}=a_{4}(I, L) \ln \mu^{2}$ in the effective action $W$ determines the one-loop beta function, this term describes renormalization of the one-loop logarithmic divergences appearing in the theory.

\section{Applications}

\subsection{Free energy for boson fields}

A finite temperature field theory is defined in Euclidean space, since for boson fields one has to impose periodic boundary conditions on imaginary time coordinate (antiperiodic boundary conditions for fermion fields respectively). A partition function is defined by

$$
Z(\beta)=\operatorname{Tr} e^{-\beta H},
$$


where $H$ is a hamiltonian of the problem and $\beta=\hbar / T$. Let us choose the lagrangian density $\rho$ in the form

$$
\rho=-\frac{\partial^{2}}{\partial \tau^{2}}+L
$$

where $\tau$ is an imaginary time coordinate and $L$ is a three dimensional spatial part of the density in the form (11). The free energy of the system is defined by

$$
F(\beta)=-\frac{\hbar}{\beta} \ln Z(\beta)=-\frac{\hbar}{\beta} \ln \left(N_{\beta} \int D \phi \exp \left(-\int_{0}^{\beta} d \tau \int d^{3} x \phi \rho \phi\right)\right),
$$

the integration is over all periodic fields satisfying $\phi(\tau+\beta)=\phi(\tau)\left(N_{\beta}\right.$ is a normalization coefficient). As a result the eigenfunctions of $\rho$ have the form $\exp \left(i \tau \omega_{n}\right) \phi_{\lambda}$, where $\omega_{n}=2 \pi n / \beta$ and $L \phi_{\lambda}=\lambda \phi_{\lambda}$. The free energy is thus equal to [1]

$$
F=\frac{\hbar}{2 \beta} \sum_{n=-\infty}^{+\infty} \sum_{\lambda} \ln \frac{\left(\omega_{n}^{2}+\lambda\right)}{\mu^{2}}=-\left.\frac{\hbar}{2 \beta} \frac{\partial}{\partial s}\left(\mu^{2 s} \zeta(s)\right)\right|_{s=0},
$$

where we introduced $\zeta$-function

$$
\zeta(s)=\sum_{n=-\infty}^{+\infty} \sum_{\lambda}\left(\omega_{n}^{2}+\lambda\right)^{-s}
$$

and the parameter $\mu$ with a mass dimensionality in order to make the argument of the logarithm dimensionless (also see a previous section).

Then it is convenient to use the formula

$$
\zeta(s)=\frac{1}{\Gamma(s)} \int_{0}^{+\infty} d t t^{s-1} \sum_{n=-\infty}^{+\infty} \sum_{\lambda} e^{-t\left(\omega_{n}^{2}+\lambda\right)},
$$

and separate $n=0$ and other terms in the sum. For $n \neq 0$ terms we substitute the heat kernel expansion for the operator $L$ at small $t$

$$
\sum_{\lambda} e^{-\lambda t}=K(I ; L ; t) \simeq \sum_{k=0}^{\infty} a_{k}(I, L) t^{(k-3) / 2}
$$

and perform $t$ integration, then we arrive at the high temperature expansion $(\beta \rightarrow 0)$ for the free energy $F$ :

$$
\begin{aligned}
& F / \hbar=-\frac{1}{2 \beta} \zeta_{L}^{\prime}(0)-\frac{1}{2 \beta} \zeta_{L}(0) \ln \left(\mu^{2}\right)+(4 \pi)^{3 / 2}\left[-\frac{a_{0}}{\beta^{4}} \frac{\pi^{2}}{90}-\frac{a_{1}}{\beta^{3}} \frac{\zeta_{R}(3)}{4 \pi^{3 / 2}}-\frac{a_{2}}{\beta^{2}} \frac{1}{24}\right. \\
& +\frac{a_{3}}{\beta} \frac{1}{(4 \pi)^{3 / 2}} \ln \left(\frac{\beta \mu}{2 \pi}\right)-\frac{a_{4}}{16 \pi^{2}}\left(\gamma+\ln \frac{\beta \mu}{2 \pi}\right) \\
& \left.-\sum_{n \geq 5} \frac{a_{n}}{\beta^{4-n}} \frac{(2 \pi)^{3 / 2-n}}{2 \sqrt{2}} \Gamma\left(\frac{n-3}{2}\right) \zeta_{R}(n-3)\right] .
\end{aligned}
$$


Here $a_{k} \equiv a_{k}(I, L), \zeta_{R}(s)=\sum_{n=1}^{+\infty} n^{-s}$ is a Riemann zeta function, $\zeta_{L}(s)=$ $\sum_{\lambda} \lambda^{-s}$ is a zeta function of an operator $L, \gamma$ is the Euler constant. The first two terms on the r.h.s. of (31) follow from the $n=0$ term.

The term

$$
-\frac{(4 \pi)^{3 / 2} \hbar a_{0}}{\beta^{4}} \frac{\pi^{2}}{90}=-V \frac{\operatorname{tr} I}{\hbar^{3}} \frac{\pi^{2}}{90} T^{4}
$$

is the leading high temperature contribution to the free energy.

The classical limit terms due to the equality $\zeta_{L}(0)=a_{3}$ can be rewritten as follows:

$$
T\left(-\frac{1}{2} \zeta_{L}^{\prime}(0)+\zeta_{L}(0) \ln \frac{\hbar}{2 \pi T}\right)=T \sum_{\lambda} \ln \frac{\hbar \sqrt{\lambda}}{2 \pi T} .
$$

The terms on the 1.h.s. of (33) yield a renormalized value of the terms on the r.h.s. of (33), since the sum on the righthandsight is generally divergent when the number of modes is infinite.

The term with $a_{4}$ determines the part of the free energy that appears due to one-loop logarithmic divergences and thus it depends on the dimensional parameter $\mu$ as in the zero temperature case.

\subsection{Chiral anomaly in four dimensions for MIT bag boundary conditions}

Consider the Dirac operator on an $n$-dimensional Riemannian manifold

$$
\widehat{D}=\gamma^{\mu}\left(\partial_{\mu}+V_{\mu}+i A_{\mu} \gamma^{5}-\frac{1}{8}\left[\gamma_{\rho}, \gamma_{\sigma}\right] \sigma_{\mu}^{[\rho \sigma]}\right)
$$

in external vector $V_{\mu}$ and axial vector $A_{\mu}$ fields. We suppose that $V_{\mu}$ and $A_{\mu}$ are anti-hermitian matrices in the space of some representation of the gauge group. $\sigma_{\mu}^{[\rho \sigma]}$ is the spin-connection ${ }^{1}$.

The Dirac operator transforms covariantly under infinitesimal local gauge transformations (the local gauge transformation is $\widehat{D} \rightarrow \exp (-\lambda) \widehat{D} \exp (\lambda))$ :

$$
\begin{aligned}
& \delta_{\lambda} A_{\mu}=\left[A_{\mu}, \lambda\right] \\
& \delta_{\lambda} V_{\mu}=\partial_{\mu} \lambda+\left[V_{\mu}, \lambda\right] \\
& \widehat{D} \rightarrow \widehat{D}+[\widehat{D}, \lambda]
\end{aligned}
$$

and under infinitesimal local chiral transformations (the local chiral transformation is $\left.\widehat{D} \rightarrow \exp \left(i \varphi \gamma_{5}\right) \widehat{D} \exp \left(i \varphi \gamma_{5}\right)\right)$ :

$$
\begin{aligned}
& \tilde{\delta}_{\varphi} A_{\mu}=\partial_{\mu} \varphi+\left[V_{\mu}, \varphi\right], \\
& \tilde{\delta}_{\varphi} V_{\mu}=-\left[A_{\mu}, \varphi\right], \\
& \widehat{D} \rightarrow \widehat{D}+i\left\{\widehat{D}, \gamma^{5} \varphi\right\} .
\end{aligned}
$$

\footnotetext{
${ }^{1}$ The spin-connection must be included even on a flat manifold if the coordinates are not Cartesian.
} 
The parameters $\lambda$ and $\varphi$ are anti-hermitian matrices.

We adopt the zeta-function regularization and write the one-loop effective action for Dirac fermions at zero temperature as ${ }^{2}$

$$
W=-\ln \operatorname{det} \widehat{D}=-\frac{1}{2} \ln \operatorname{det} \widehat{D}^{2}=\frac{1}{2} \zeta_{\widehat{D}^{2}}^{\prime}(0)+\frac{1}{2} \ln \left(\mu^{2}\right) \zeta_{\widehat{D}^{2}}(0),
$$

where

$$
\zeta_{\widehat{D}^{2}}(s)=\operatorname{Tr}\left(\widehat{D}^{-2 s}\right),
$$

prime denotes differentiation with respect to $s$, and $\operatorname{Tr}$ is the functional trace.

The following identity holds:

$$
\zeta_{A}(s)=\operatorname{Tr} A^{-s} \Rightarrow \delta \zeta_{A}(s)=-s \operatorname{Tr}\left((\delta A) A^{-s-1}\right) .
$$

Due to the identity (39)

$$
\delta_{\lambda} \zeta_{\widehat{D}^{2}}(s)=-\left(2 s \operatorname{Tr}\left([\widehat{D}, \lambda] \widehat{D}^{-2 s-1}\right)\right)=-2 s\left(\operatorname{Tr}\left(\left[\widehat{D}^{-2 s}, \lambda\right]\right)\right)=0,
$$

so the effective action (37) is gauge invariant, $\delta_{\lambda} W=0$.

The chiral anomaly is by definition equal to the variation of $W$ under an infinitesimal chiral transformation. Using (39) we obtain:

$$
\tilde{\delta}_{\varphi} \zeta_{\widehat{D}^{2}}(s)=-\left(2 i s \operatorname{Tr}\left(\left\{\widehat{D}, \gamma^{5} \varphi\right\} \widehat{D}^{-2 s-1}\right)\right)=-4 i s\left(\operatorname{Tr}\left(\gamma^{5} \varphi \widehat{D}^{-2 s}\right)\right)
$$

and the anomaly reads

$$
\mathcal{A}:=\tilde{\delta}_{\varphi} W=\frac{1}{2} \tilde{\delta}_{\varphi} \zeta_{\widehat{D}^{2}}^{\prime}(0)=-\left.2 \operatorname{Tr}\left(i \gamma^{5} \varphi \widehat{D}^{-2 s}\right)\right|_{s=0} .
$$

The heat kernel is related to the zeta function by the Mellin transformation:

$$
\operatorname{Tr}\left(i \gamma^{5} \varphi \widehat{D}^{-2 s}\right)=\Gamma(s)^{-1} \int_{0}^{\infty} d t t^{s-1} K\left(i \gamma^{5} \varphi, \widehat{D}^{2}, t\right) .
$$

In particular, after the substitution of the heat kernel expansion (13) into the formula (43) we obtain

$$
\mathcal{A}=-2 a_{n}\left(i \gamma^{5} \varphi, \widehat{D}^{2}\right)
$$

The same expression for the anomaly follows also from the Fujikawa approach [5].

We impose local boundary conditions:

$$
\left.\Pi_{-} \psi\right|_{\partial M}=0, \quad \Pi_{-}=\frac{1}{2}\left(1-\gamma^{5} \gamma_{n}\right),
$$

\footnotetext{
${ }^{2}$ The one-loop effective action is proportional to Planck constant $\hbar$, in what following we put $\hbar=1$.
} 
which are nothing else than a Euclidean version of the MIT bag boundary conditions $[8]$. For these boundary conditions $\Pi_{-}^{\dagger}=\Pi_{-}$, and the normal component of the fermion current $\psi^{\dagger} \gamma_{n} \psi$ vanishes on the boundary.

Since $\widehat{D}$ is a first order differential operator it was enough to fix the boundary conditions (45) on a half of the components. To proceed with a second order operator $L=\widehat{D}^{2}$ we need boundary conditions on the remaining components as well. They are defined by the consistency condition [12]:

$$
\left.\Pi_{-} \widehat{D} \psi\right|_{\partial M}=0
$$

which is equivalent to the Robin boundary condition

$$
\left.\left(\nabla_{n}+S\right) \Pi_{+} \psi\right|_{\partial M}=0, \quad \Pi_{+}=\frac{1}{2}\left(1+\gamma^{5} \gamma_{n}\right)
$$

with

$$
S=-\frac{1}{2} \Pi_{+} L_{a a}
$$

In the paper [7] the following expression for a coefficient $a_{4}(Q, L)$ with an hermitian matrix valued function $Q$ and conditions (5),$L_{a b}=0$ (flat boundaries), $S=0$ was obtained:

$$
\begin{aligned}
a_{4}(Q, L)= & \frac{1}{360}(4 \pi)^{-n / 2}\left\{\int _ { M } d ^ { n } x \sqrt { g } \operatorname { t r } \left\{Q \left(60 E_{; \mu}{ }^{\mu}+60 R E+180 E^{2}\right.\right.\right. \\
& \left.\left.+30 \Omega_{\mu \nu} \Omega^{\mu \nu}+12 R_{; \mu}{ }^{\mu}+5 R^{2}-2 R_{\mu \nu} R^{\mu \nu}+2 R_{\mu \nu \rho \sigma} R^{\mu \nu \rho \sigma}\right)\right\} \\
& +\int_{\partial M} d^{n-1} x \sqrt{h} \operatorname{tr}\left\{Q \left\{30 E_{; n}+30 \chi E_{; n} \chi+90 \chi E_{; n}+90 E_{; n} \chi\right.\right. \\
& +18 \chi \chi_{: a} \Omega_{a n}+12 \chi_{: a} \Omega_{a n} \chi+18 \Omega_{a n} \chi \chi_{: a}-12 \chi \Omega_{a n} \chi_{: a} \\
& \left.+6\left[\chi \Omega_{a n} \chi, \chi_{: a}\right]+54\left[\chi_{: a}, \Omega_{a n}\right]+30\left[\chi, \Omega_{a n: a}\right]+12 R_{; n}+30 \chi R_{; n}\right\}+ \\
& +Q_{; n}(-30 E+30 \chi E \chi+90 \chi E+90 E \chi- \\
& \left.\left.\left.-18 \chi_{: a} \chi_{: a}+30 \chi_{: a a}-6 \chi_{: a} \chi_{: a} \chi+30 \chi R\right)+30 \chi Q_{; \mu}{ }^{\mu n}\right\}\right\} .
\end{aligned}
$$

To obtain the chiral anomaly in four dimensions ${ }^{3}$ with MIT bag boundary conditions one has to calculate the coefficient $a_{4}(Q, L)$ (49) with $L=\widehat{D}^{2}, Q=$ $i \gamma^{5} \phi$ and substitute it into (44). We define $V_{\mu \nu}=\partial_{\mu} V_{\nu}-\partial_{\nu} V_{\mu}+\left[V_{\mu}, V_{\nu}\right], A_{\mu \nu}=$ $D_{\mu} A_{\nu}-D_{\nu} A_{\mu}, D_{\mu} A_{\nu}=\partial_{\mu} A_{\nu}-\Gamma_{\mu \nu}^{\rho} A_{\rho}+\left[V_{\mu}, A_{\nu}\right]$. The anomaly contains two contributions:

$$
\mathcal{A}=\mathcal{A}_{V}+\mathcal{A}_{b}
$$

\footnotetext{
${ }^{3}$ In two dimensions $(n=2)$ the boundary part of the chiral anomaly with MIT bag boundary conditions is equal to zero 7 .
} 
In the volume part

$$
\begin{aligned}
& \mathcal{A}_{V}=\frac{-1}{180(2 \pi)^{2}} \int_{M} d^{4} x \sqrt{g} \operatorname{tr} \varphi\left(-120\left[D_{\mu} V^{\mu \nu}, A_{\nu}\right]\right. \\
& \quad+60\left[D_{\mu} A_{\nu}, V^{\mu \nu}\right]-60 D_{\mu} D^{\mu} D_{\nu} A^{\nu}+120\left\{\left\{D_{\mu} A_{\nu}, A^{\nu}\right\}, A^{\mu}\right\} \\
& \quad+60\left\{D_{\mu} A^{\mu}, A_{\nu} A^{\nu}\right\}+120 A_{\mu} D_{\nu} A^{\nu} A^{\mu}+30\left[\left[A_{\mu}, A_{\nu}\right], A^{\mu \nu}\right] \\
& \quad+\epsilon_{\mu \nu \rho \sigma}\left\{-45 i V^{\mu \nu} V^{\rho \sigma}+15 i A^{\mu \nu} A^{\rho \sigma}-30 i\left(V^{\mu \nu} A^{\rho} A^{\sigma}+A^{\mu} A^{\nu} V^{\rho \sigma}\right)\right. \\
&\left.-120 i A^{\mu} V^{\nu \rho} A^{\sigma}+60 i A^{\mu} A^{\nu} A^{\rho} A^{\sigma}\right\}-60\left(D_{\sigma} A_{\nu}\right) R^{\nu \sigma}+30\left(D_{\mu} A^{\mu}\right) R \\
&\left.-\frac{15 i}{8} \epsilon_{\mu \nu \rho \sigma} R^{\mu \nu}{ }_{\eta \theta} R^{\rho \sigma \eta \theta}\right)
\end{aligned}
$$

only the $D A-R$ terms seem to be new [7] (for flat space it can be found e.g. in 6] ).

The boundary part

$$
\begin{aligned}
\mathcal{A}_{b}=\frac{-1}{180(2 \pi)^{2}} \int_{\partial M} d^{3} x \sqrt{h} \operatorname{tr}\left(12 i \epsilon^{a b c}\left\{A_{b}, \varphi\right\} D_{a} A_{c}\right. \\
+24\left\{\varphi, A^{a}\right\}\left\{A_{a}, A_{n}\right\}-60\left[A^{a}, \varphi\right]\left(V_{n a}-\left[A_{n}, A_{a}\right]\right) \\
\left.+60\left(D_{n} \varphi\right) D_{\mu} A^{\mu}\right)
\end{aligned}
$$

is new [7. It has been derived under the two restrictions: $S=0$ and $L_{a b}=0$. Note, that in the present context, the first condition $(S=0)$ actually follows from the second one $\left(L_{a b}=0\right)$ due to (48).

\section{Acknowledgements}

V.M. thanks the organizers of the VIII Training Course in the Physics of Correlated Electron Systems and High-Tc Superconductors for partial support and hospitality in Vietri sul Mare (Salerno). V.M. is grateful to Dr. Adolfo Avella for his help and efforts that made the visit to Vietri possible. V.M. thanks Dmitri Vassilevich for his suggestions during the preparation of the paper. This work has been supported by the Ostpartnerschaften program of Leipzig University.

\section{References}

[1] D. V. Vassilevich, Phys.Rept. 388, 279 (2003) arXiv:hep-th/0306138.

[2] J.S.Dowker and G.Kennedy, J.Phys.A 11, 895 (1978).

[3] S.L.Adler, Phys. Rev. 177, 2496 (1969); J.S.Bell and R.Jackiw, Nuovo Cim. A 60, 47 (1969). 
[4] R. A. Bertlmann, "Anomalies In Quantum Field Theory", Clarendon Press, Oxford, 1996.

[5] K. Fujikawa, Phys. Rev. Lett. 42, 1195 (1979).

[6] A. A. Andrianov and L. Bonora, Nucl. Phys. B 233, 232 (1984).

[7] V.N.Marachevsky and D.V.Vassilevich, Nucl. Phys. B 677, 535 (2004) [arXiv: hep-th/0309019].

[8] A. Chodos, R. L. Jaffe, K. Johnson, C. B. Thorn and V. F. Weisskopf, Phys. Rev. D 9, 3471 (1974);

A. Chodos, R. L. Jaffe, K. Johnson and C. B. Thorn, Phys. Rev. D 10, 2599 (1974);

T. DeGrand, R. L. Jaffe, K. Johnson and J. E. Kiskis, Phys. Rev. D 12, 2060 (1975) .

[9] P. B. Gilkey, "Invariance theory, the heat equation, and the Atiyah-Singer index theorem", CRC Press, 1994.

[10] T. P. Branson and P. B. Gilkey, Commun. Part. Diff. Equat.15, 245 (1990);

D. V. Vassilevich, J. Math. Phys. 36, 3174 (1995) arXiv:gr-qc/9404052;

T. P. Branson, P. B. Gilkey, K. Kirsten and D. V. Vassilevich, Nucl. Phys. B 563, 603 (1999) arXiv:hep-th/9906144.

[11] M.Bordag, U.Mohideen, V.M.Mostepanenko, Phys.Rept. 353, 1 (2001) (pp.113 - 117).

[12] T. Branson and P. Gilkey, J. Funct. Anal. 108, 47 (1992); Differential Geom. Appl. 2, 249 (1992). 